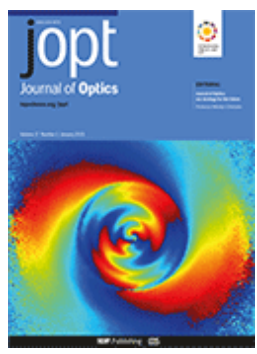

(C) 2015 IOP Publishing Ltd.

This article was published in: Journal of Optics 17, 065612 (2015)

DOI: $10.1088 / 2040-8978 / 17 / 6 / 065612$

Available online at:

http://iopscience.iop.org/2040-8986/17/6/065612 


\title{
Phase and group velocity of focused, pulsed Gaussian beams in the presence and absence of primary aberrations
}

\author{
Balázs Major ${ }^{1}$, Zoltán L. Horváth ${ }^{1}$ and Miguel A. Porras ${ }^{2}$ \\ ${ }^{1}$ Department of Optics and Quantum Electronics, University of Szeged, Dóm tér 9., \\ Szeged H-6720, Hungary \\ ${ }^{2}$ Grupo de Sistemas Complejos, Universidad Politécnica de Madrid, Rios Rosas 21, \\ Madrid ES-28003, Spain \\ E-mail: bmajor@titan.physx.u-szeged.hu
}

\begin{abstract}
This work presents a study on the phase- and group-velocity variations of focused, pulsed Gaussian beams during the propagation through the focal region along the optical axis. In aberration-free case it is discussed how the wavelength dependence of beam properties alters the group velocity, and how a chromatic aberration-like effect can arise even when focusing is performed with an element which does not have chromatic aberration. It is also examined what effects primary spherical aberration, astigmatism, coma, curvature of field and distortion along with chromatic aberration have on the phase- and group-velocity changes occurring during propagation through focus.
\end{abstract}

PACS numbers: 42.15.Fr Aberrations, 42.25.Fx Diffraction and scattering, 42.65.Re Ultrafast processes

Keywords: focusing, diffraction, primary aberrations, phase velocity, group velocity Submitted to: J. Opt. 


\section{Introduction}

The axial phase shift of spherical waves during propagation through a focus was discovered by Gouy in the end of the 19th century [1]. Since then, the generality of this effect for any kind of beams was revealed, even in the case of matter waves [2]. Just recently a spectral analogue was also demonstrated [3], and the transverse nature of Gouy phase has also been profoundly studied [4]. Still, the physical origin of this effect has been interpreted in several different ways [5-8], only a recent publication aiming to unify the diverse explanations [9]. Nevertheless, the importance of this phenomenon is incontrovertible in several fields of science, like nanophotonics [10], high-harmonic generation [11,12], light-based metrology [13], interferometry [14] and THz-spectroscopy [15].

An interesting consequence of this phase shift is that in the focal region the wave acquires a superluminal phase velocity. It has been shown, that this property is also true for the group velocity of pulsed Gaussian beams [16,17]. Similarly to the case of focusing, the on-axis change of the phase and group velocities has also been studied for a pulsed plane wave diffracted by a circular aperture [18]. These pulse-velocity properties, being immediate consequences of the Gouy phase shift, are important in several fields of non-linear optical sciences [19], especially in experiments where phasematching is crucial $[11,12,19]$. Gouy phase and its concomitant phenomena have also been shown to be relevant in several cases where few-cycle pulses are used $[13,15]$. As the carrier-envelope phase (CEP) is determined by the difference between the phase and group velocities, the variations of these latter two quantities are also significant in CEP-dependent effects [20].

Still, previous studies do not take into account intrinsic beam properties or the aberrations of the focusing system. In addition, while the focal intensity distribution in the case of aberration-distorted focusing of monochromatic beams [21-23], and the spatio-temporal shape of pulsed beams in the focal region of aberrated systems have been the subject of a wide range of studies [24-35], the effect of aberrations on the phase or the Gouy phase shift has been scarcely investigated [8,36].

In this paper the phase and group velocities of focused, pulsed Gaussian beams are studied in the presence and absence of primary aberrations. It is taken into account that Gaussian beams generally are not focused at their waist, and how this property affects the behaviour of the group velocity in the focal region. The appreciable influence of the wavelength dependence of general beam properties - like beam waist size or the distance of the beam waist and the focusing element - on the on-axis group velocity is demonstrated. This color-dependence of beam characteristics are usually not considered and not known in experiments, but shown here to exist and to be relevant in general. Along with the effect of chromatic aberration, it is investigated how primary monochromatic aberrations (spherical aberration, astigmatism, coma, curvature of field and distortion) change the on-axis phase and group velocity. The most important symmetry properties of the acquired velocity variations are summarized, 


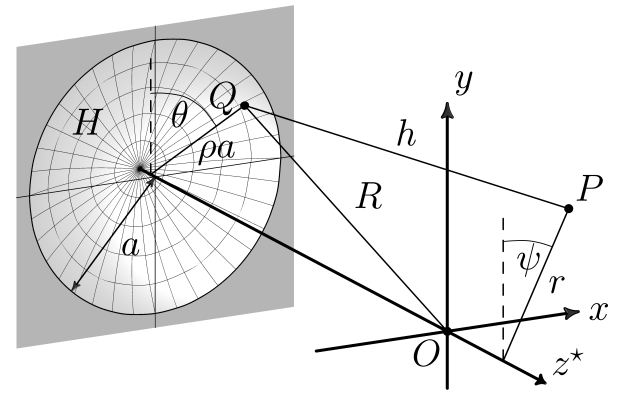

Figure 1. The notation and coordinate system used in the calculations for a monochromatic beam with frequency $\omega$.

and comparisons are drawn between the recent findings and results of related previous studies.

\section{Methods}

\subsection{Aberration-distorted focusing}

Consider a linearly polarized pulsed beam, so that the electric field can be treated as a scalar waveform. The pulsed beam can be regarded as a superposition of monochromatic beams. According to the Huygens-Fresnel principle [23], the focusing of such a monochromatic component can be treated as the propagation of a converging wave front through an aperture having radius $a$ (see Figure 1).

In an aberration-distorted case the amplitude and phase of the field of a monochromatic component in a point $P$ close to the focus can be acquired by the evaluation of the integral

$$
U(P)=-\frac{\mathrm{i}}{\lambda} \frac{\exp (-\mathrm{i} k R)}{R} \iint_{H} \frac{A \exp (\mathrm{i} k(h+\Phi))}{h} \mathrm{~d} H .
$$

In the expression above $k=2 \pi / \lambda=\omega / c$ is the wave number, $R$ is the radius of the paraxial wave front (the paraxial focal length if a plane wave is focused), $A$ is a possibly space-dependent amplitude, $h$ is the distance between the point of interest $P$ and a typical point $Q$ of the surface $H$ along which the integration is performed, and $\Phi=\Phi(\rho, \theta)$ is the so-called aberration function which represents the deviation of the wave front from the non-aberrated one, which is a spherical surface [23]. Please note that in general $\Phi$ depends on the object point chosen. But if this point is fixed, the explicit dependence can be omitted [23]. In this work we consider such cases, and further considerations about this will be discussed in a later chapter. With the assumption of an input Gaussian beam with beam diameter $2 w$ and phase front radius $R$ in the exit aperture, (1) yields [37-39]

$$
U(P)=-\frac{\mathrm{i} \omega}{2 c} \frac{A a^{2}}{R\left(z+R_{0}\right)} \exp \left(\mathrm{i} k\left(z+R_{0}-R\right)\right)
$$




$$
\times \exp \left(\mathrm{i} k \frac{r^{2}}{2\left(z+R_{0}\right)}\right) \frac{1}{\pi} I(u, v, \psi),
$$

where

$$
\begin{aligned}
& I(u, v, \psi)=\int_{0}^{1} \int_{0}^{2 \pi} \exp \left(-\kappa \rho^{2}\right) \\
& \quad \times \exp \left(\mathrm{i} k \Phi-\mathrm{i} v \rho \cos (\theta-\psi)-\mathrm{i} \frac{u}{2} \rho^{2}\right) \rho \mathrm{d} \theta \mathrm{d} \rho,
\end{aligned}
$$

and

$$
\begin{aligned}
u & =\frac{\omega}{c} \frac{a^{2}}{R} \frac{z+R_{0}-R}{z+R_{0}}=\frac{\omega}{c} \frac{a^{2}}{R} \frac{z^{\star}}{z+R_{0}}, \\
v & =\frac{\omega}{c} \frac{a}{z+R_{0}} r .
\end{aligned}
$$

In the expressions above $z^{\star}$ is the axial coordinate from the focus at frequency $\omega$, placed at distance $R=R(\omega)$ from the vertice of the surface $H$, and $z$ is the axial coordinate from the focus at $\omega_{0}$, placed at distance $R_{0}=R\left(\omega_{0}\right)$ (see Figure 1). The beam size at the exit pupil $w=w(\omega)$ can also be frequency dependent, and related to it another dimensionless parameter introduced here is the truncation coefficient $\kappa=(a / w)^{2}$ [37], the effect of which for monochromatic case has been studied in detail in [38]. Other parameters and a short summary of the notation can be seen in Figure 1.

For the subsequent calculations the important quantity is the phase $\varphi(z, r, \psi)=$ $\arg [U(P)]$ of the focused field, which is

$$
\begin{aligned}
\varphi(z, r, \psi)=\frac{\omega}{c}(z & \left.+R_{0}-R\right)+\frac{\omega}{c} \frac{r^{2}}{2\left(z+R_{0}\right)}-\frac{\pi}{2} \\
& +\arg [I(u, v, \psi)] .
\end{aligned}
$$

\subsection{The phase and group velocities}

The main goal of this paper is to investigate how the phase and group velocities of a focused, pulsed Gaussian beam change during propagation in the focal region. As is known [23], the phase velocity of general time-harmonic electric field can be obtained from the space-dependent phase $\varphi=\varphi(z, r, \phi)$ of the wave using the simple expression

$$
v_{\mathrm{p}}=\frac{\omega}{|\nabla \varphi|} .
$$

The gradient operator

$$
\nabla \varphi=\boldsymbol{u}_{r} \partial_{r} \varphi+\boldsymbol{u}_{\phi} \frac{1}{r} \partial_{\phi} \varphi+\boldsymbol{u}_{z} \partial_{z} \varphi
$$

will be used in the cylindrical form, as the considered physical problems are cylindrically symmetric, and the formula for the space-dependent phase is expressed in such coordinate system formed by the three unit vectors $\boldsymbol{u}_{r}, \boldsymbol{u}_{\phi}$ and $\boldsymbol{u}_{z}$. Note however, that the usual definition of the azimuthal coordinate $\phi$ differs in (8) from the one used in (3), named $\psi$. As $\psi+\phi=\pi / 2$ (see Figure 1), the derivative with respect to $\phi$ can be replaced by the derivative with respect to $\psi$ according to $\partial_{\psi} \varphi=-\partial_{\phi} \varphi$. 
The group velocity of a waveform with central (or mean) frequency $\omega_{0}$, by definition, is

$$
v_{\mathrm{g}}=\frac{1}{\left|\nabla\left(\left.\partial_{\omega} \varphi\right|_{\omega=\omega_{0}}\right)\right|},
$$

and its exact evaluation will be detailed in a later section. It is important to add here that the above expression for the group velocity is strictly valid only if the spectral bandwidth $\Delta \omega$ of the studied radiation is much smaller then central frequency $\omega_{0}$, that is $\Delta \omega / \omega_{0} \ll 1$.

\section{Results and discussion}

\subsection{On-axis phase velocity in aberration-free focusing of pulsed Gaussian beams}

First let us consider the case of aberration-free focusing, which means $\Phi \equiv 0$ in (3). The on-axis phase in this case has already been derived in [40] for the two limiting cases considering the truncation of the Gaussian beam. In the case of strong truncation $(\kappa \rightarrow 0)$ it has been shown that the physical meaning of the on-axis group velocity is doubtful [40], due to the discontinuities of the phase on axis [41]. For this reason, this case is excluded from the following analytical analysis of aberration-free focusing, and only the other limit, the situation of an untruncated beam $(\kappa \rightarrow \infty)$ will be discussed. In the $\kappa \rightarrow \infty$ limit the on-axis $(r=0)$ phase $\varphi(z)$ of the field $U(P)$ simplifies to the expression [40]

$$
\varphi(z)=\frac{\omega}{c} z^{\star}-\operatorname{atan}(\omega T(z, \omega))-\frac{\pi}{2},
$$

where a new variable has been introduced, which is

$$
T(z, \omega)=\frac{w^{2}}{2 c R} \frac{z+R_{0}-R}{z+R_{0}} .
$$

By evaluating the phase velocity of the wave having angular frequency $\omega$ using (7) and (10) one can arrive to the expression

$$
\frac{v_{\mathrm{p}}}{c}=\frac{1+\omega^{2} T^{2}}{1+\omega^{2} T^{2}-c T^{\prime}},
$$

where prime denotes partial derivative with respect to coordinate $z$, and the arguments of $T$ have been omitted for conciseness. This simple relation of the on-axis phase velocity to the speed of light in vacuum $c$ shows that $v_{\mathrm{p}} \geq c$, as

$$
T^{\prime}=\frac{w^{2}}{2 c} \frac{1}{\left(z+R_{0}\right)^{2}} \geq 0 .
$$

It can also be simply deduced that the phase velocity reaches its maximum in the diffraction focus, as the position of maximum can be found to be

$$
z_{v_{\mathrm{p}, \max }}=R-R_{0}-\frac{R}{1+\pi^{2} N_{w}^{2}},
$$

where $N_{w}=w^{2} / \lambda R$ is the Fresnel number of the Gaussian beam at the focusing element. It can be seen, that for large Fresnel numbers the differences between the phase velocities 
of different monochromatic components of a pulse appear due to a chromatic aberrationlike effect in focusing (see later that the wavelength dependence of $R$ is not necessarily caused by the chromatic aberration of the focusing element), while for small Fresnel numbers the well-known focal shift also appears [42], which alters the variation of the on-axis phase velocity as well.

\subsection{On-axis group velocity in aberration-free focusing of pulsed Gaussian beams}

The group velocity of the focused pulse with carrier $\omega_{0}$ can be obtained with simple algebra using (9) and (10). Taking into account that both the beam size $w=w(\omega)$ at the focusing element and the reference sphere radius $R=R(\omega)$ can depend on frequency, the ratio of the group velocity and the speed of light in vacuum can be found to be

$$
\begin{aligned}
\left(\frac{v_{\mathrm{g}}}{c}\right)^{-1} & =1-g \frac{\left(1-\omega_{0}^{2} T_{0}^{2}\right) c T_{0}^{\prime}}{\left(1+\omega_{0}^{2} T_{0}^{2}\right)^{2}} \\
& +\gamma \frac{\left(w_{0}^{2} / R_{0}\right) \omega_{0}^{2} T_{0} T_{0}^{\prime}}{\left(1+\omega_{0}^{2} T_{0}^{2}\right)^{2}}
\end{aligned}
$$

In the previous expression two dimensionless parameters were introduced, which characterize the beam at the focusing element and the paraxial wave front leaving it. The first parameter

$$
g=1+2 \frac{\dot{w}_{0}}{w_{0}} \omega_{0}
$$

characterizes the wavelength dependence of the beam size at the aperture, and

$$
\gamma=-\frac{\dot{R}_{0}}{R_{0}} \omega_{0}
$$

describes the frequency dependence of the radius of the wave front filling the exit aperture (see [43], but note the different normalization). In the two expressions above (and throughout the paper) the dot sign indicates partial derivative with respect to angular frequency and subscript zero means evaluation at $\omega_{0}$.

It can be easily seen, that (15) is a generalized form of the expressions formulated in [17] and [40]. Assuming constant beam waists located just in front of the focusing element without chromatic aberration (i.e. $g=1$ and $\gamma=0$ ), (15) reduces to (29) in [40], and simplifies to (35) of [17] in the case of $\gamma=0$.

The analytical expression (15) has the advantage that the effect of the two parameters $g$ and $\gamma$ can be analyzed independently (see Figure 2). As it can bee seen in Figure 2(a), for large Fresnel numbers $N_{w}$ the non-zero value of $g$ induces an on-axis variation of the group velocity which is an even function of $z$. This is by the reason of the focused Rayleigh range variation inherent to the focused beam size variation, which is the dominant effect in the case of $g \neq 0$ and $\gamma=0$. So, as the Gouy phase shift occurs in regions of different lengths for different wavelengths, the group velocity variation is symmetric with respect to the geometrical focus. If $\gamma \neq 0$ and $g=0$, however, the components are focused to different points in space, which decisively determine the onaxis group velocity changes. For this reason the group velocity variation in this case 

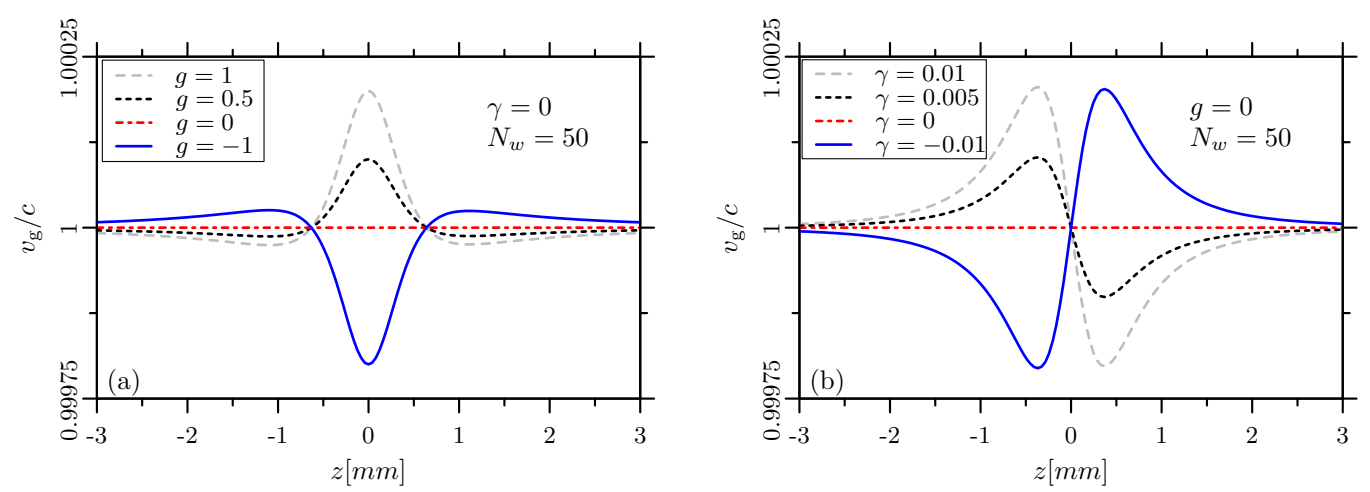

Figure 2. The on-axis group velocity variation of a $w_{0}=2 \mathrm{~mm}, \lambda=800 \mathrm{~nm}$ pulsed Gaussian beam in case of negligible truncation. (a) The change of on-axis group velocity for different values of $g$, which characterizes the wavelength dependence of the beam spot size at the focusing element (see (15)). (b) The change of on-axis group velocity for different values of $\gamma$, which characterizes the wavelength dependence of the radius of the paraxial wave front (see (15)).

is an odd function of $z$ (Figure 2(b)). For $\gamma>0$ (the usual chromatic aberration of lenses), blue components are focused closer to the focusing element, which appears as group velocity increase in front of the geometrical focal point of the carrier wave, and a decrease behind it (as discussed in [44]).

In case of small Fresnel numbers, the well-known focal shift occurs, and an asymmetric change in the group velocity variation appears (see [40] for the $g \neq 0$ and $\gamma=0$ case). An interesting situation is that with $g=0$ and $\gamma=0$ the group velocity is constant during propagation on-axis. Based on later considerations, this can be achieved only by focusing an isodiffracting pulsed Gaussian beam at its waist with a focusing element that has no chromatic aberration.

\subsection{Dependence of $g$ and $\gamma$ parameters on the source beam and the focusing system properties}

In previous studies $[17,40]$, only those cases were considered when the position of the beam waist is exactly at the focusing element, and this position is the same for all frequencies of the pulsed beam. However, in general, this assumption is not true, and the distance between the waist of the source beam is far from the element used for focusing. So, for more accurate description, it has to be considered how the parameters $g$ and $\gamma$ appearing in (15) depend on parameters of the source beam and the focusing element.

Let us consider a pulsed Gaussian beam, which has a wavelength-dependent beam waist radius $s=s(\omega)$ (inherently involves a wavelength dependent Rayleigh range $\left.L=L(\omega)=\omega s^{2} /(2 c)\right)$. We also allow the possibility, that the exact distance $d=d(\omega)$ of the beam waist and the focusing element can also depend on the frequency of the radiation (see Figure 3). Using the well-known expressions for the propagation of 


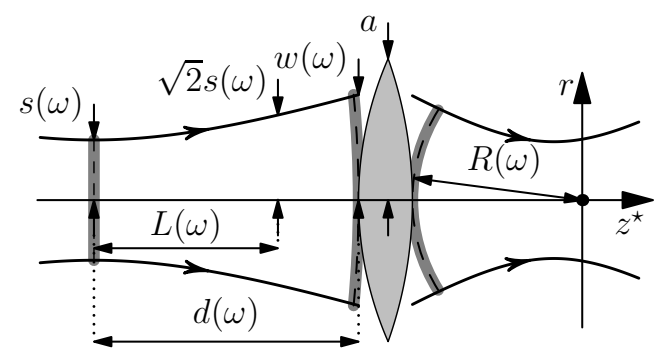

Figure 3. Figure describing the beam parameters.

Gaussian beams [45], one can express the $g$ parameter as

$$
g=g_{s}+\left(\varepsilon_{s}-g_{s}\right) \frac{2 \xi^{2}}{1+\xi^{2}} .
$$

In the previous expression the following dimensionless parameters were introduced:

$$
g_{s}=1+2 \frac{\dot{s}_{0}}{s_{0}} \omega_{0}=\frac{\dot{L}_{0}}{L_{0}} \omega_{0}
$$

is similar to (16) and describes the frequency dependence of the source beam's Rayleigh range (waist radius), and

$$
\varepsilon_{s}=\frac{\dot{d}_{0}}{d_{0}} \omega_{0}
$$

corresponds to the wavelength dependence of the source beam's waist position. The variable $\xi=d_{0} / L_{0}$ is the dimensionless distance of the beam waist and the focusing element at $\omega_{0}$. This means that $g$ depends only on two properties of the source beam: the wavelength dependences of the beam waist size and of the waist distance from the focusing element. If the beam waist is at the focusing element, that is $\xi=0$, then $g=g_{s}$ and only the source-beam waist-size variation is relevant.

By looking at the other parameter, $\gamma$, it has to be taken into account that not just chromatic aberration of the lens have to be considered, but the wavelength-dependent parameters of the source beam also have an effect on it, as the radius of paraxial wave front $R$ depends on the radius of the input spherical wave. Knowing that in the paraxial approximation a focusing element with focal length $f$ transforms a spherical wave with radius $R_{s}$ into a spherical wave with radius $R$ according to $1 / R=1 / f-1 / R_{s}$, the $\gamma$ parameter can be found to be

$$
\begin{aligned}
\gamma=\gamma_{f} & \frac{\xi+1 / \xi}{\xi+1 / \xi-\chi} \\
& +\frac{\chi}{1+\xi^{2}} \frac{\left(\xi^{2}-1\right) \varepsilon_{s}+2 g_{s}}{\xi+1 / \xi-\chi} .
\end{aligned}
$$

The new parameters introduced here are

$$
\gamma_{f}=-\frac{\dot{f_{0}}}{f_{0}} \omega_{0}
$$


describing the chromatic aberration of the focusing element, and $\chi=f_{0} / L_{0}$ is a dimensionless form of the focal length. It can be seen in (21) that the chromatic aberration of the focusing element is the most relevant term, and the beam properties become important when $\chi \sim 1$, so in situations when Debye approximation looses its validity.

These results show that the group velocity in the case of aberration-free focusing of pulsed Gaussian beams can be unambiguously described by the knowledge of the source beam's waist size (or Rayleigh range), the waist's distance from the focusing element, the focusing element's focal length, and the frequency dependence of the previous three quantities.

To understand the values of the parameters in Figure 2, we emphasize that $g_{s}=1,0,-1$ correspond to wavelength-independent beam waist size, Rayleigh range and divergence, respectively [46]. The case $g_{s}=0$ is the natural configuration of beams from a stable open laser resonators [47]. For $\gamma_{f}$ of the focusing element, the value $\gamma_{f}=0.01$ is within the order of magnitude for the chromatic aberration of common commercial biconvex lenses. Regarding the sign of $\gamma_{f}$, positive values correspond to normal dispersion, and negative values to anomalous dispersion of the lens material. Equation (21) also shows that $\gamma \neq 0$ can appear also for mirrors, since $\gamma_{f} \neq 0$ (longitudinal chromatic aberration of the focusing element) is not necessary for $\gamma \neq 0$, as in the case of a beam that is not focused at its waist.

\subsection{Phase and group velocities in case of aberration-distorted focusing of pulsed Gaussian beams}

In the following the phase and group velocity of the carrier wave of a pulsed Gaussian beam will be discussed in the presence of aberrations. To clearly distinguish the effects of aberrations from those coming from truncation, the considerations are restricted to weakly truncated beams. We will also limit our considerations to propagation along the optical axis to simplify the treatment, and as relevant changes appear on-axis even in such aberration-distorted cases, when the optical axis is considered as axis of symmetry in imaging. The aberration function $\Phi(\rho, \theta)$ for a single aberration will be written in the form

$$
\Phi_{l n m}(\rho, \theta)=A_{l n m} \rho^{n} \cos ^{m} \theta=\mu \lambda_{0} \rho^{n} \cos ^{m} \theta,
$$

so the extent of the aberration is described in the units of the carrier wavelength. As it was noted in a previous chapter, the aberration function $\Phi$ generally depends on the object point chosen (explicitly given by parameter $Y_{1}^{\star}$ in [23]). However, in a wave optical description the shape of the wave front is relevant, which is described in this case by $\mu$, and naturally depends on $Y_{1}^{\star}$. Certainly, different selections of $Y_{1}^{\star}$ can yield the same $\mu$. For a particular $Y_{1}^{\star}$ in an experimental realization the value of $\mu$ can be calculated, e.g. with ray tracing, and the results presented here can be applied accordingly.

If aberrations are present, the phase- and group-velocity variation can not be treated analytically in general. The factor in (6) that necessitates the numerical evaluation of 
velocities is $\varphi_{I}=\arg [I(u, v, \psi)]$, as this needs the determination of the argument of a complex number, which is acquired by the evaluation of an integral, then the calculation of its derivative.

In the aberration-distorted case the on-axis phase velocity of the carrier wave can be calculated according to

$$
\begin{aligned}
& \frac{v_{\mathrm{p}}(z)}{c}=\left|\frac{\left.c \nabla \varphi\right|_{\substack{r=0 \\
\omega=\omega_{0}}}}{\omega_{0}}\right|^{-1},
\end{aligned}
$$

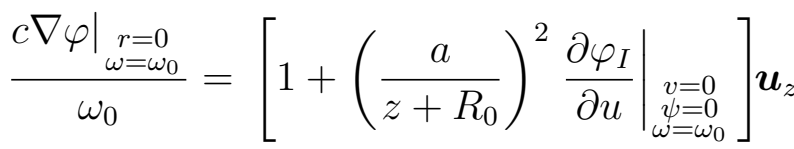

$$
\begin{aligned}
& +\left[\left.\frac{a}{z+R_{0}} \frac{\partial \varphi_{I}}{\partial v}\right|_{\substack{v=0 \\
y=0 \\
w=\omega_{0}}}\right] \boldsymbol{u}_{r} .
\end{aligned}
$$

The on-axis group velocity can be obtained by

$$
\begin{aligned}
& \frac{v_{\mathrm{g}}(z)}{c}=\left|c \nabla\left(\frac{\partial \varphi}{\partial \omega} \mid \begin{array}{c}
r=0 \\
\omega=\omega_{0}
\end{array}\right)\right|^{-1}, \\
& c \nabla\left(\left.\frac{\partial \varphi}{\partial \omega}\right|_{\substack{r=0 \\
\omega=\omega_{0}}}\right)= {\left[1+\left.\omega_{0}\left(\frac{a}{z+R_{0}}\right)^{2} \frac{\partial^{2} \varphi_{I}}{\partial u \partial \omega}\right|_{\substack{v=0 \\
y=0 \\
\omega=\omega_{0}}}\right] \boldsymbol{u}_{z} } \\
&+ {\left[\left.\omega_{0} \frac{a}{z+R_{0}} \frac{\partial^{2} \varphi_{I}}{\partial v \partial \omega}\right|_{\substack{v=0 \\
\psi=0 \\
w=\omega_{0}}}\right] \boldsymbol{u}_{r} . }
\end{aligned}
$$

In the expressions above the $\boldsymbol{u}_{\phi}$ component of the gradient is omitted, because due to the arbitrary nature of the azimuthal coordinate in case of on-axis points, the azimuthal component is irrelevant. It appears in the calculations in the form that for $\psi=0$ the $\boldsymbol{u}_{\psi}$ component disappears, for other $\psi \neq 0$ values necessarily the vectorial sum of $\boldsymbol{u}_{r}$ and $\boldsymbol{u}_{\psi}$ components result in the same vector as for the $\psi=0$ case. For this reason, the calculations were carried out with $\psi=0$.

It is added here that for aberrations that are characterized by an even value of $m$ the $\boldsymbol{u}_{r}$ component of the velocities is also zero, so the phase-and group-velocity vectors are parallel with the optical axis. The possible wavelength variation of the aberration [48, 49], which generally almost always present, is not considered here. In the following, the considerations will be limited to primary aberrations appearing independently, but any combinations of primary (or higher order) aberrations could be treated using the velocity expression above.

It is also noted that for large Fresnel numbers (when the Debye approximation is appropriate [50]) the on-axis phase velocity can be acquired only by changing (24), explicitly by replacing the factor $a /\left(z+R_{0}\right)$ by $a / R_{0}$ in every occurrence. A similar simple scaling is also true for the on-axis group velocity in the case of $\gamma=0$. However, it has been shown that in case of aberration-distorted focusing, the validity of Debye approximation may require more than the large value of the Fresnel number $[51,52]$. 

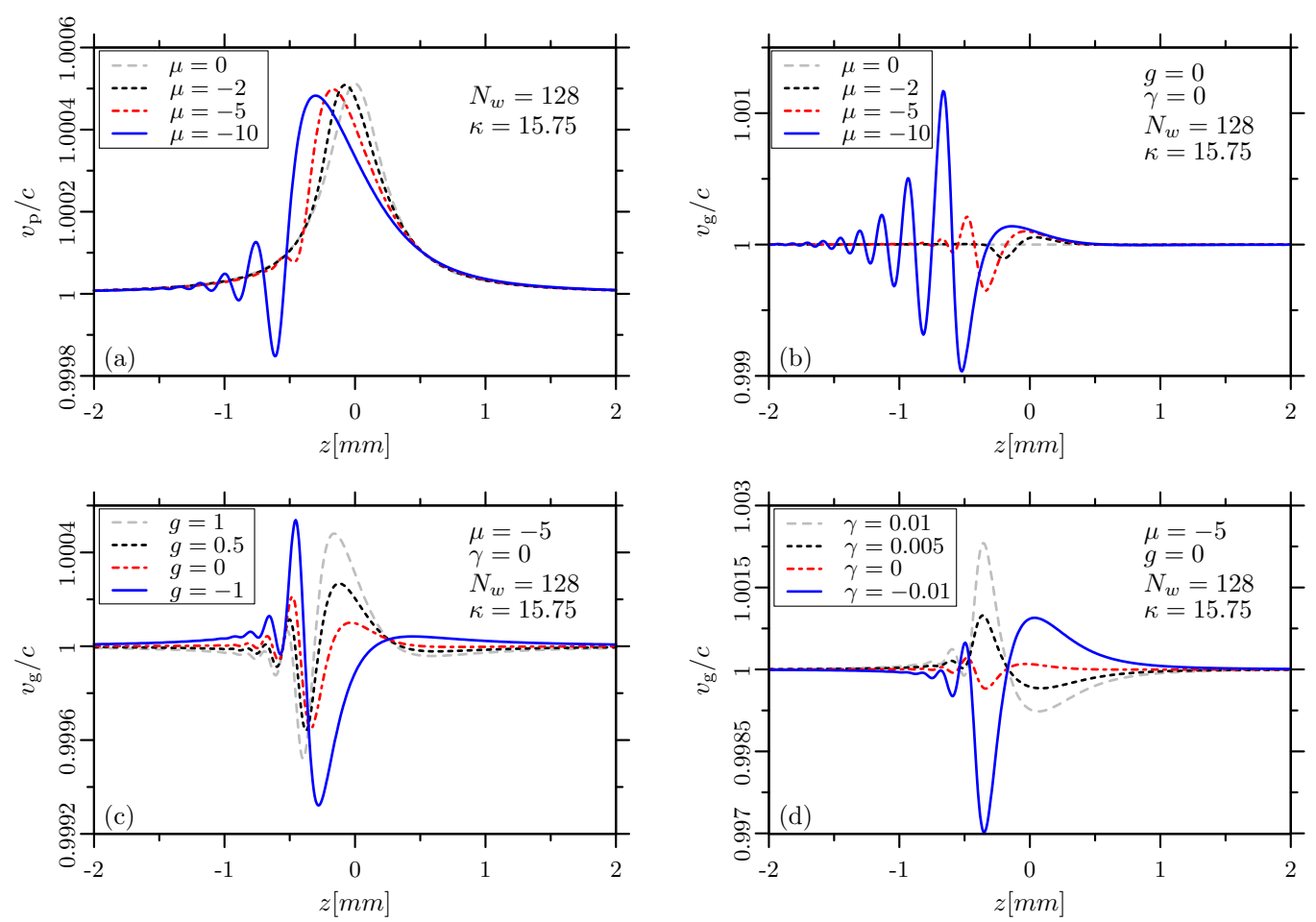

Figure 4. The effect of primary spherical aberration on the on-axis phase and group velocities of a pulsed Gaussian beam with $\lambda_{0}=800 \mathrm{~nm}$ carrier wavelength and $w_{0}=3.2 \mathrm{~mm}$ beam radius at the focusing element which has one inch aperture. The radius of the paraxial wave front leaving the focusing element is $R_{0}=100 \mathrm{~mm}$. The Rayleigh range of focused beam is $\approx 250 \mu \mathrm{m}$. (a) Phase velocities for different amounts of primary spherical aberration. (b) Group velocities for different amounts of primary spherical aberration. (c) Group velocities for different values of $g$. (d) Group velocities for different values of $\gamma$.

It will also been shown later in an example, how the other requisite for the Debye approximation to be valid, introduced in [51], appears in aberration-distorted focusing.

\subsection{Primary spherical aberration}

Primary spherical aberration is known to be present in the case of focusing with spherical lenses or spherical mirrors, even when the optical axis of the focusing element and the direction of beam propagation coincide. The aberration function of primary spherical aberration is indexed by $l=m=0$ and $n=4$, so has the form $[23,34]$

$$
\Phi_{040}(\rho, \theta)=\mu \lambda_{0} \rho^{4} .
$$

The fourth power dependence on the dimensionless radial coordinate $\rho$ nicely shows that for rays propagating close to the optical axis, the effect of this aberration is small. For homogeneous illumination with a monochromatic beam it has been recently shown, that spherical aberration has a relevant effect on the phase properties in the vicinity of the focus, and alters the well-known Gouy phase [36]. 
Figure 4 shows the on-axis phase- and group-velocity variation in the presence of primary aberration of a focused, pulsed beam for particular cases. The aberration coefficient $\mu$ has been chosen to be negative, because for general focusing systems it is always negative (this means that rays farther from axis will be focused to a point closer to the focusing element), and because it has been shown recently that the field in Debye approximation possesses a mirror-image symmetry with respect to $u=0$ considering the sign of the aberration coefficient [36]. This means that only the $u \leftrightarrow-u$ and $\gamma \leftrightarrow-\gamma$ exchanges have to be made if $\mu \leftrightarrow-\mu$ and the Debye approximation is valid. Note that there would be slight differences between the two cases if the Debye approximation is not valid due to the $z$ dependent scaling in (24) and (25). For the most common cases, the spherical aberration would cause variations in the phase and group velocities before the focal point $(z<0)$, as expected (see Figure $4(\mathrm{a})$ and $(\mathrm{b})$ ).

\subsection{Primary astigmatism}

Primary astigmatism can appear, for example, when spherical mirrors are used for offaxis focusing, even in the cases which can be considered as paraxial. The aberration function of primary astigmatism is indexed by $l=0$ and $m=n=2$, so has the form $[23,34]$

$$
\Phi_{022}(\rho, \theta)=\mu \lambda_{0} \rho^{2} \cos ^{2} \theta .
$$

Primary astigmatism arises when skew rays are present in the imaging, and in this case meridional and sagittal rays are focused to two distinct points. It has been shown both theoretically and experimentally that the effect of this double focal point is that the total $\pi$ phase shift of Gouy happens in two steps of $\pi / 2$ shift $[8,53]$.

The two focal points become more distinct with increasing aberration (see Figure 5 (a) and (b)), and phase and group velocities reach local maximums at these two points. The astigmatism is unique type of primary aberrations as for $g=0$ and $\gamma=0$ the group velocity is constant during on-axis propagation. This is by the reason of the symmetry in $\theta$ of the aberration function $(m=2)$. Note that the phase and group velocity variations have a similar symmetry in case of primary astigmatism as they have with primary spherical aberration (exchange $\mu \leftrightarrow-\mu$ means only exchanges $u \leftrightarrow-u$ and $\gamma \leftrightarrow-\gamma)$. This is due to the even value of $n$.

\subsection{Primary coma}

Primary coma is present if skew rays which are filling a bigger part of the aperture are involved in the focusing, and appears also for non-perfectly adjusted parabolic mirrors. The aberration function of primary coma is indexed by $l=0, n=3$ and $m=1$, so has the form $[23,34]$

$$
\Phi_{031}(\rho, \theta)=\mu \lambda_{0} \rho^{3} \cos \theta .
$$

Primary coma lengthens the area where the Gouy phase shift occurs, it produces a region where the phase changes almost linearly with distance, which appears as a longer 

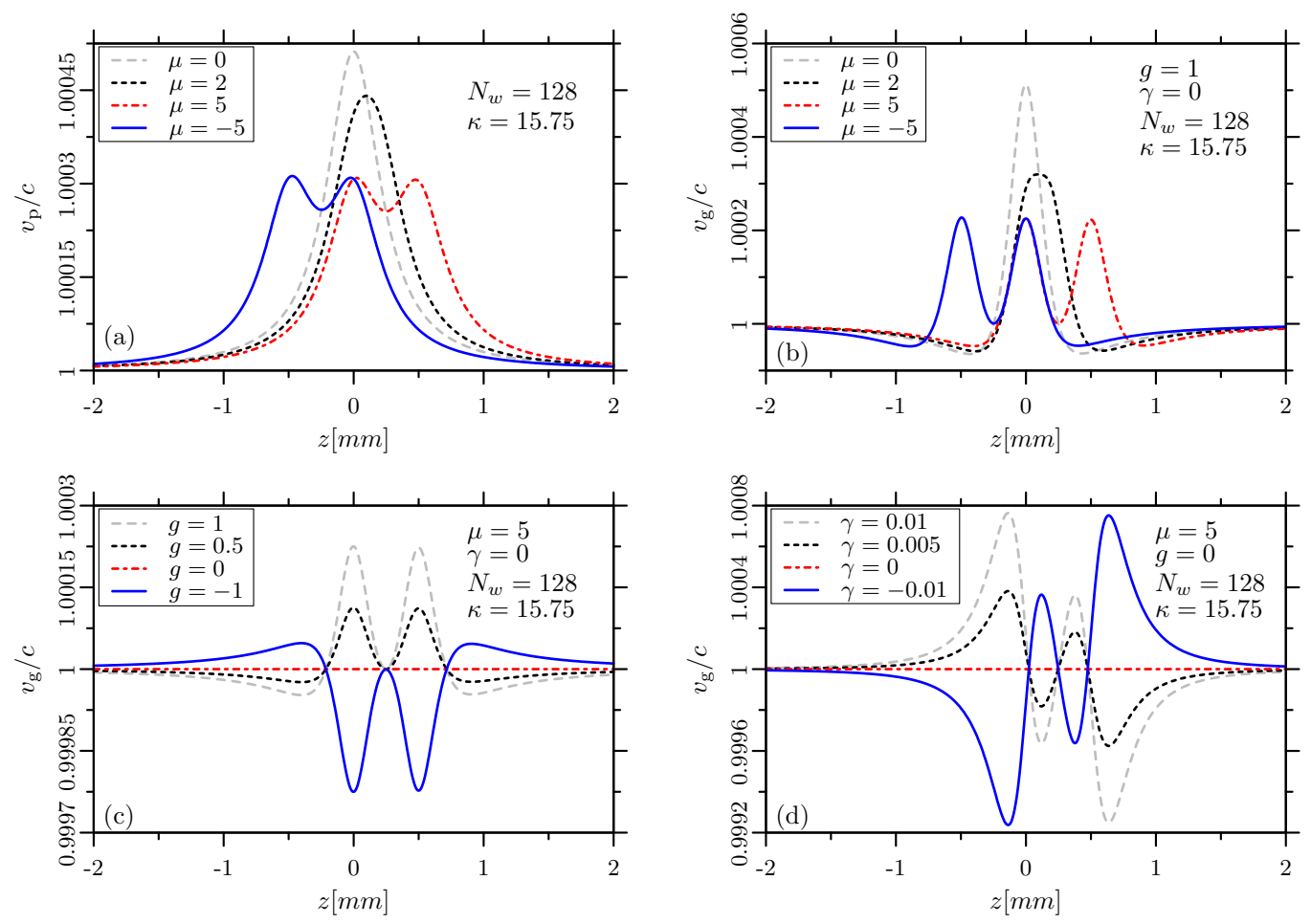

Figure 5. The effect of primary astigmatism on the on-axis phase and group velocities of a pulsed Gaussian beam with $\lambda_{0}=800 \mathrm{~nm}$ carrier wavelength and $w_{0}=3.2 \mathrm{~mm}$ beam radius at the focusing element which has one inch aperture. The radius of the paraxial wave front leaving the focusing element is $R_{0}=100 \mathrm{~mm}$. The Rayleigh range of focused beam is $\approx 250 \mu \mathrm{m}$. (a) Phase velocities for different amounts of primary astigmatism. (b) Group velocities for different amounts of primary astigmatism. (c) Group velocities for different values of $g$. (d) Group velocities for different values of $\gamma$.

space where phase velocity has a constant $v_{0}^{(p)}>c$ value (see Figure 6). Note, that in the $\gamma=0$ case the group velocity variation is symmetric with respect to $u=0$ in the Debye approximation. It is also important, that the direction of phase velocities, and due to this reason the direction of group velocity, is not parallel to the $z$ axis, and has a radial component. This stand for every primary aberration where $m$ is odd. Another property of aberrations with odd $m$ is that in the Debye approximation the on-axis variation of the amplitude of the phase and group velocities are identical when $|\mu|$ is the same.

\subsection{Primary curvature of field}

Primary curvature of field is mostly mentioned in imaging related problems, as this aberration causes the general experience that a flat object, which is normal to the optical axis, can not be imaged sharply in every point to a plane (image plane). The aberration function of primary curvature of field is indexed by $l=1, n=2$ and $m=0$, so has the form $[23,34]$

$$
\Phi_{120}(\rho, \theta)=\mu \lambda_{0} \rho^{2} .
$$



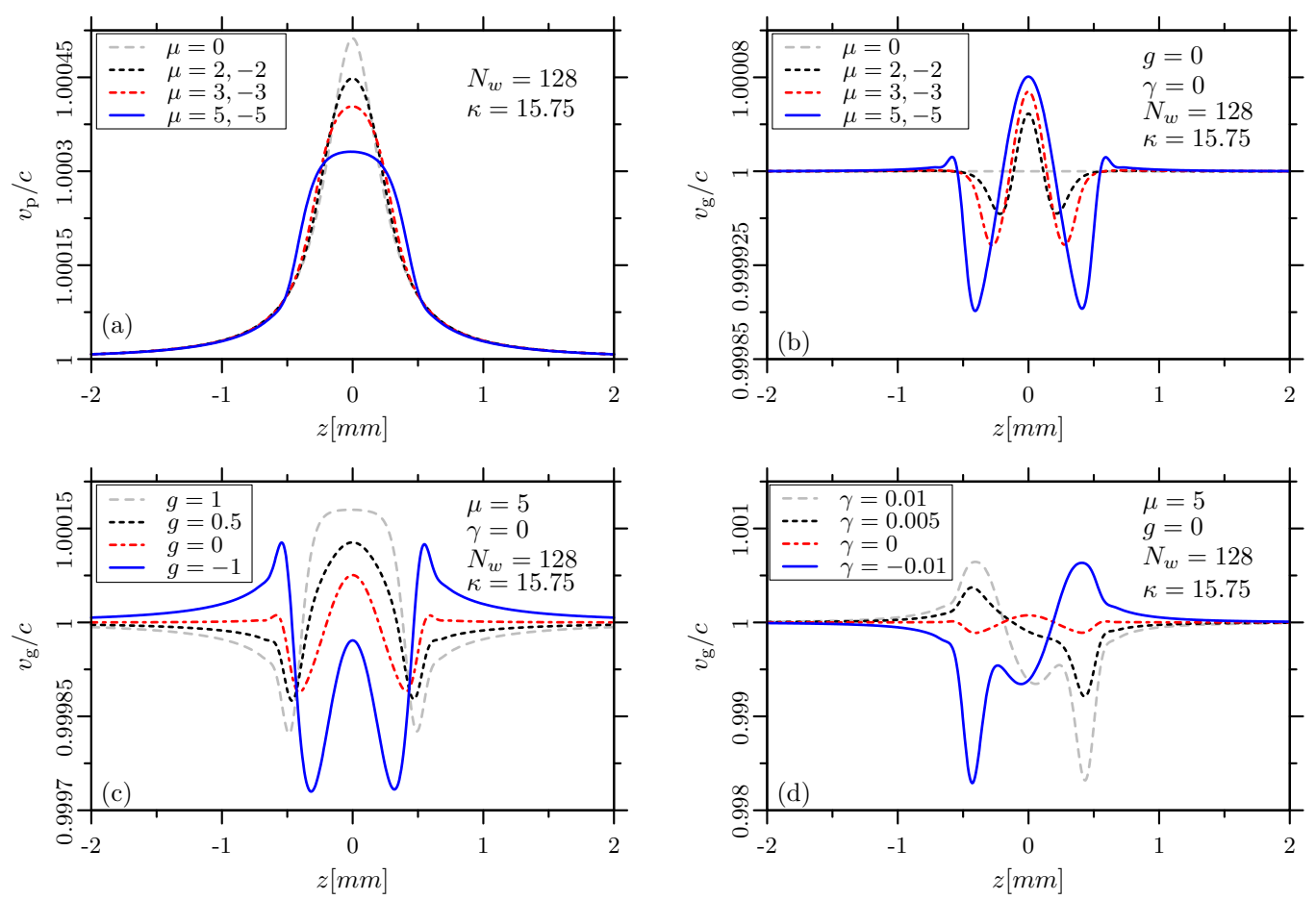

Figure 6. The effect of primary coma on the on-axis phase and group velocities of a pulsed Gaussian beam with $\lambda_{0}=800 \mathrm{~nm}$ carrier wavelength and $w_{0}=3.2 \mathrm{~mm}$ beam radius at the focusing element which has one inch aperture. The radius of the paraxial wave front leaving the focusing element is $R_{0}=100 \mathrm{~mm}$. The Rayleigh range of focused beam is $\approx 250 \mu \mathrm{m}$. (a) Phase velocities for different amounts of primary coma. (b) Group velocities for different amounts of primary coma. (c) Group velocities for different values of $g$. (d) Group velocities for different values of $\gamma$.
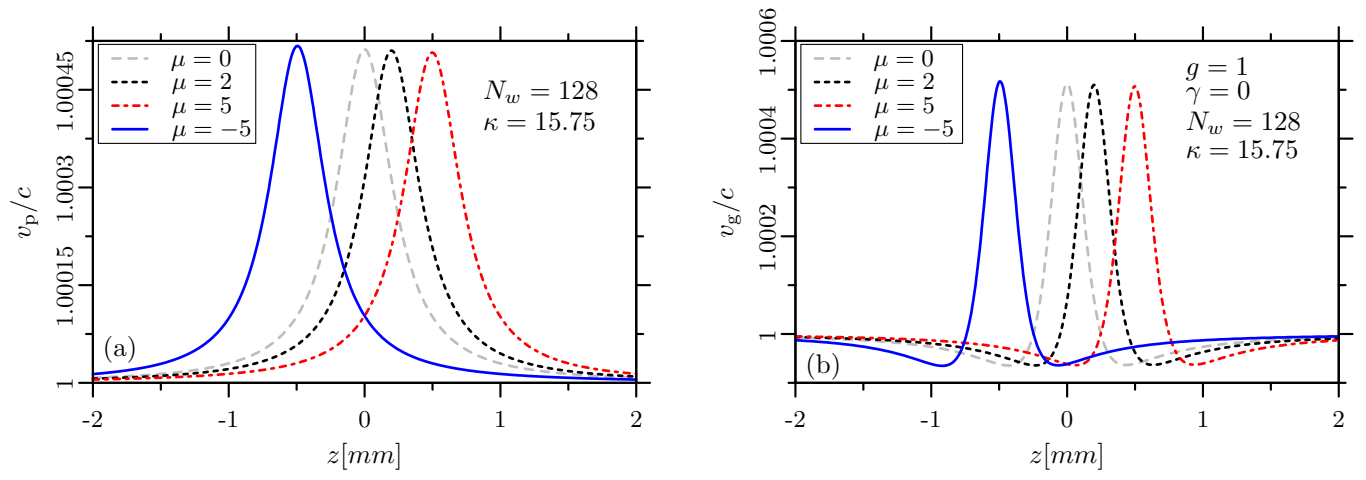

Figure 7. The effect of primary curvature of field on the on-axis phase and group velocities of a pulsed Gaussian beam with $\lambda_{0}=800 \mathrm{~nm}$ carrier wavelength and $w_{0}=3.2 \mathrm{~mm}$ beam radius at the focusing element which has one inch aperture. The radius of the paraxial wave front leaving the focusing element is $R_{0}=100 \mathrm{~mm}$. The Rayleigh range of focused beam is $\approx 250 \mu \mathrm{m}$. (a) Phase velocities for different amounts of spherical aberration. (b) Group velocities for different amounts of spherical aberration. 

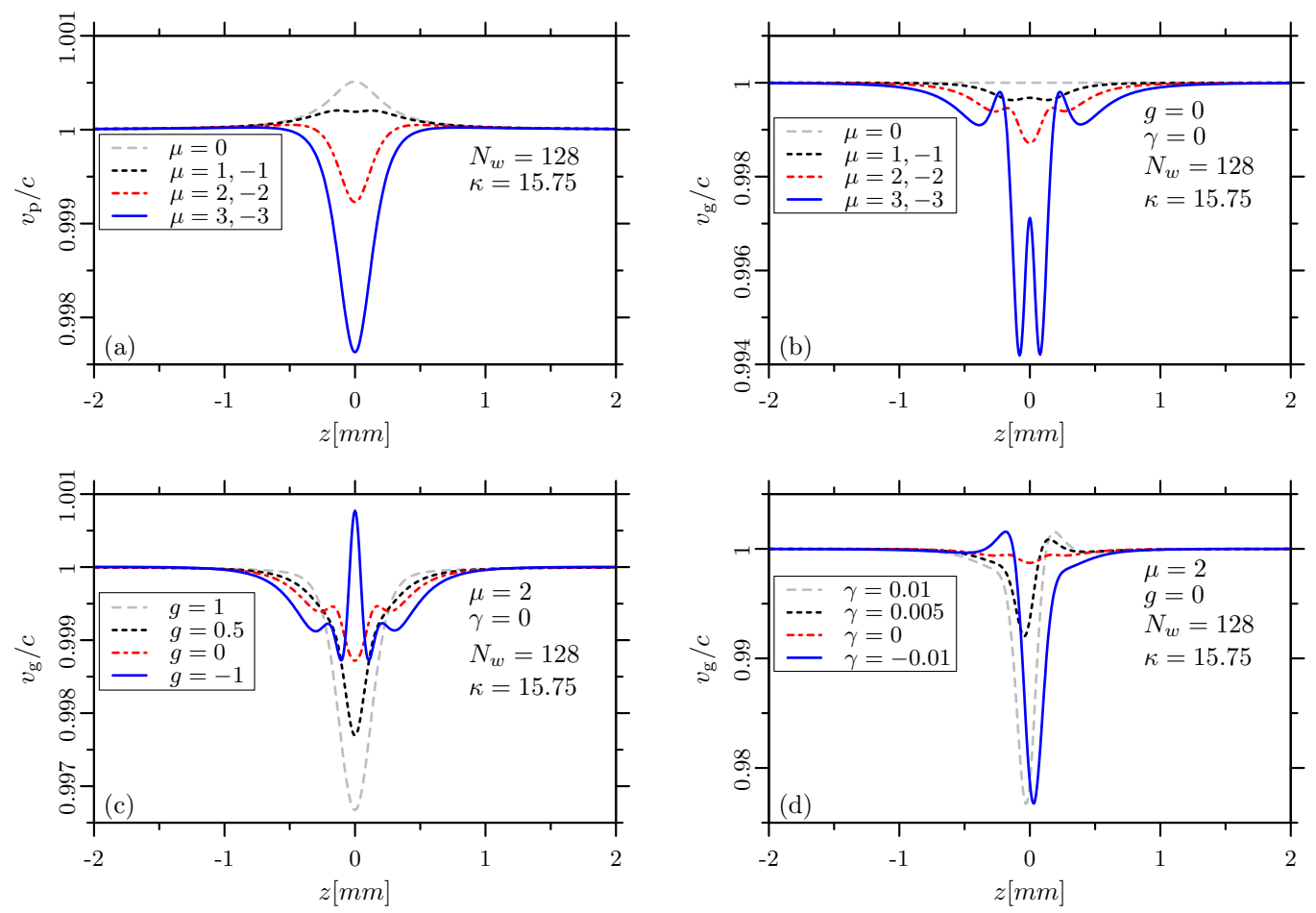

Figure 8. The effect of primary distortion on the on-axis phase and group velocities of a pulsed Gaussian beam with $\lambda_{0}=800 \mathrm{~nm}$ carrier wavelength and $w_{0}=3.2 \mathrm{~mm}$ beam radius at the focusing element which has one inch aperture. The radius of the paraxial wave front leaving the focusing element is $R_{0}=100 \mathrm{~mm}$. The Rayleigh range of focused beam is $\approx 250 \mu \mathrm{m}$. (a) Phase velocities for different amounts of primary distortion. (b) Group velocities for different amounts of primary distortion. (c) Group velocities for different values of $g$. (d) Group velocities for different values of $\gamma$.

As is known [34], the effect of primary curvature of field is just a spatial and temporal shift with respect to the aberration-free case, if the Debye approximation is applicable. This can be easily seen, as by substituting (29) into (3), the integral acquires a shift along $u$ by $u_{\text {shift }}=\mu 4 \pi$. Note however, as mentioned earlier, the validity of Debye approximation has more strict requirements in the case of aberration-distorted focusing $[51,52]$. This appears as a coordinate-dependent scaling of phase and group velocities, as it can be seen in Figure 7 (a) and (b). This is a nice example of the requirement of $u \ll 2 \pi N_{w}$ for the Debye approximation to be valid in this case [51].

\subsection{Primary distortion}

Primary distortion is also best-known from imaging, especially the two main types of it: barrel and pincushion type distortion. The aberration function of primary distortion is indexed by $l=n=m=1$, so has the form $[23,34]$

$$
\Phi_{111}(\rho, \theta)=\mu \lambda_{0} \rho \cos \theta .
$$


Examining the graphs plotted in Figure 8 it is at first surprising that primary distortion produces a $v_{0}^{(p)}<c$ on-axis in a larger part of the focal region, and this variation is symmetrical with respect to $u=0$ (see Figure 8 (a)). It also makes it peculiar that it gives rise to a phase and group velocity difference compared to the speed of light in vacuum (meaning $v / c-1$ ) that is an order of magnitude larger than the effect of other aberrations of same amount (same value of $\mu$ ), as it can be seen in Figure 8. This is, however, easy to understand if it is considered that primary distortion means a spatial shift in the $y$ direction in Debye approximation [34]. It has been shown, that the amount of the spatial shift depends on the amount of aberration [34]. It is also known that along the radial coordinate $r$ the value of the phase and group velocities decrease, become subluminal far from axis, and this subluminal velocity change is larger in amplitude then the on-axis superluminal variation [16]. So, evaluating the on-axis phase and group velocities in the presence of primary distortion is identical to the calculation of the variation of the same properties in an aberration-free situation along a line which is parallel to the optical axis and lies in the $y-z$ plane. Like the other aberrations characterized by odd $m$, primary distortion produces the same phase or group velocity variation for an aberration of the same amplitude, but opposite sign. Like in the case of primary coma, both the phase- and the group-velocity vectors have a component, that is not parallel to the optical axis $z$.

\section{Conclusions}

In this work it was studied how the phase and group velocities of focused, pulsed Gaussian beams change while the pulse propagates through the focal region along the optical axis. It was taken into account for the first time, to our knowledge, how the wavelength dependence of beam parameters alter the phase and group velocities. These wavelength dependencies are experimentally relevant and can not be avoided. It was shown that due to these effects a chromatic aberration-like distortion can appear even when the focusing is performed with an optical element without chromatic aberration, like a mirror. The formulas presented here for the aberration-free case unify the findings of previous studies presented in this topic.

The effects of primary aberrations, that is spherical aberration, astigmatism, coma, curvature of field and distortion on the usual propagation-dependent changes of phase and group velocities have been studied, for the first time, which are again experimentally unavoidable sources of distortion. For this reason the simulation results presented here are crucial in experiments where not just the intensity distribution, but the phase variation is also important. Such research topics are mainly focused on, but not limited to, nonlinear optical effects, where phase matching is essential. So the findings presented here aim to provide help for studies like high harmonic generation or phenomena based on optical rectification (e.g. terahertz radiation generation). The proper knowledge of the phase and group velocity changes are also important for CEP-dependent effects, as the variation of CEP originates in the changes of the two velocities. 


\section{Acknowledgments}

The work of B.M. was supported by the European Union and the State of Hungary, co-financed by the European Social Fund in the framework of TÁMOP 4.2.4.A/2-11-12012-0001 'National Excellence Program'. M.A.P. acknowledges support from Projects No. MTM2012-39101-C02-01 and No. FIS2013-41709-P of the Spanish Ministerio de Economía y Competitividad.

\section{References}

[1] Gouy L G 1890 C. R. Acad. Sci. Paris 1101251

[2] Petersen T C, Paganin D M, Weyland M, Simula T P, Eastwood S A and Morgan M J 2013 Phys. Rev. A: At., Mol., Opt. Phys. 88043803

[3] Andresen E R, Finot C, Oron D and Rigneault H 2013 Phys. Rev. Lett. 110143902

[4] Davis B S and Kaplan L 2013 J. Opt. 15075706

[5] Boyd R W 1980 J. Opt. Soc. Am. 70 877-880

[6] Simon R and Mukunda N 1993 Phys. Rev. Lett. 70(7) 880-883

[7] Feng S and Winful H G 2001 Opt. Lett. 26 485-487

[8] Visser T and Wolf E 2010 Opt. Commun. 283 3371-3375

[9] Petersen T C, Paganin D M, Weyland M, Simula T P, Eastwood S A and Morgan M J 2014 Phys. Rev. A: At., Mol., Opt. Phys. 89063801

[10] Kim M S, Naqavi A, Scharf T, Weible K J, Vlkel R, Rockstuhl C and Herzig H P 2013 J. Opt. 15 105708

[11] Balcou P, Saliéres P, L'Huillier A and Lewenstein M 1997 Phys. Rev. A: At., Mol., Opt. Phys. 55 $3204-3210$

[12] Popmintchev T, Chen M C, Arpin P, Murnane M M and Kapteyn H C 2010 Nature Photon. 4 $822-832$

[13] Coddington I, Swann W C, Nenadovic L and Newbury N R 2009 Nature Photon. 3 351-356

[14] Pang X, Fischer D G and Visser T D 2012 J. Opt. Soc. Am. A 29 989-993

[15] Ahmed S, Savolainen J and Hamm P 2014 Opt. Express 22 4256-4266

[16] Horváth Z L, Vinkó J, Bor Z and von der Linde D 1996 Appl. Phys. B: Lasers Opt. 63 481-484

[17] Porras M A, Gonzalo I and Mondello A 2003 Phys. Rev. E: Stat., Nonlinear, Soft Matter Phys. 67(6) 066604

[18] Zhou G, Wang S, Zhao D and Xu J 2003 Opt. Commun. 224 205-211

[19] Boyd R W 2008 Nonlinear Optics 3rd ed (Academic Press)

[20] Xu R, Bai Y, Song L, Liu P, Li R and Xu Z 2013 Appl. Phys. Lett. 103061111

[21] Kant R 1993 J. Mod. Opt. 40 2293-2310

[22] Kant R 1995 J. Mod. Opt. 42 299-320

[23] Born M and Wolf E 1999 Principles of Optics 7th ed (Cambridge University Press)

[24] Kempe M and Rudolph W 1993 Phys. Rev. A: At., Mol., Opt. Phys. 48 4721-4729

[25] Mattei G and Gil M 1999 Appl. Opt. 38 1058-1064

[26] Marti-López L, Mendoza-Yero O and de Campos J A R 2001 J. Opt. Soc. Am. A 18 1348-1356

[27] Zalvidea D and Sicre E E 2003 J. Opt. A-Pure Appl. Op. 5 S310

[28] Bowlan P, Gabolde P and Trebino R 2007 Opt. Express 15 10219-10230

[29] Estrada-Silva F C, Garduno-Mejía J, Rosete-Aguilar M, Román-Moreno C J and Ortega-Martínez R 2009 Appl. Opt. 48 4723-4734

[30] Mecseki K, Kovács A P and Horváth Z L 2010 AIP Conf. Proc. 1228 190-196

[31] Gonzalez-Galicia M A, Garduno-Mejia J, Rosete-Aguilar M, Bruce N C and Ortega-Martinez R 2011 J. Opt. Soc. Am. A 28 1990-1994 
[32] Gonzalez-Galicia M A, Rosete-Aguilar M, Garduno-Mejia J, Bruce N C and Ortega-Martinez R 2011 J. Opt. Soc. Am. A 28 1979-1989

[33] García-Martínez L, Rosete-Aguilar M and Garduno-Mejia J 2012 Appl. Opt. 51 306-315

[34] Horváth Z L, Major B, Kovács A P and Bor Z 2013 J. Opt. Soc. Am. B 30 1853-1863

[35] Sun B, Salter P S and Booth M J 2014 J. Opt. Soc. Am. A 31 765-772

[36] Pang X, Fischer D G and Visser T D 2014 Opt. Lett. 39 88-91

[37] Li Y and Wolf E 1982 Opt. Commun. 42 151-156

[38] Horváth Z L and Bor Z 2003 Opt. Commun. 222 51-68

[39] Bor Z and Horváth Z 1992 Opt. Commun. 94 249-258

[40] Horváth Z L and Bor Z 1999 Phys. Rev. E: Stat., Nonlinear, Soft Matter Phys. 60(2) 2337-2346

[41] Linfoot E H and Wolf E 1956 Proc. Phys. Soc. B 69823

[42] Li Y and Wolf E 1981 Opt. Commun. 39 211-215

[43] Porras M A, Horvath Z L and Major B 2012 Appl. Phys. B: Lasers Opt. 108(3) 521-531

[44] Porras M A, Major B and Horvath Z L 2012 J. Opt. Soc. Am. B 29 3271-3276

[45] Saleh B E A and Teich M C 1991 Fundamentals of Photonics 1st ed (John Wiley \& Sons)

[46] Porras M A 2009 Opt. Lett. 34 1546-1548

[47] Feng S, Winful H G and Hellwarth R W 1999 Phys. Rev. E: Stat., Nonlinear, Soft Matter Phys. 59(4) $4630-4649$

[48] Gillman L M 1919 Trans. Opt. Soc. 21 53-64

[49] Weinstein W 1950 Br. J. Appl. Phys. 1 67-73

[50] Wolf E and Li Y 1981 Opt. Commun. 39 205-210

[51] Sheppard C J R 2000 Opt. Lett. 25 1660-1662

[52] Sheppard C J R 2013 Opt. Lett. 38 1074-1076

[53] Rolland J P, Thompson K P, Lee K S, Tamkin J, Schmid T and Wolf E 2012 Appl. Opt. 51 2902-2908 